\title{
Concurrent optimization of dimensions and tolerances on structures and mechanisms
}

\author{
Antonio Armillotta ${ }^{1}$ (D) \\ Received: 9 July 2020 / Accepted: 28 October 2020 / Published online: 7 November 2020 \\ (C) The Author(s) 2020
}

\begin{abstract}
The paper deals with a problem of robust optimization of mechanical assemblies, which combines the allocation of tolerances with the selection of dimensional parameters. The two tasks are carried out together with the aim of minimizing the manufacturing cost and the variation on an assembly-level functional characteristic. The problem is addressed in the specific context of planar linkages used in structures and mechanisms. The proposed solution is based on an optimality condition involving both tolerances and dimensions, which allows to define a joint optimization problem avoiding the need of two sequential optimization phases. The condition is developed with the method of Lagrange multipliers using an expanded formulation of the reciprocal power cost-tolerance function. The optimal tolerances depend on the stackup coefficients of the output characteristic, which are calculated with a tolerance analysis method based on a static analogy. The procedure is demonstrated on two examples to illustrate some application details and discuss potential advantages and limitations.
\end{abstract}

Keywords Tolerance allocation $\cdot$ Parameter design $\cdot$ Robust optimization $\cdot$ Planar linkages $\cdot$ Cost-tolerance function $\cdot$ Tolerance analysis

\section{Introduction}

The parts of a mechanical assembly are manufactured with random deviations from their nominal geometry. Once the parts are connected, the geometric errors stack up through the contact features between them. As a result, an assembly dimension (i.e., the relative position between features of different parts) may also deviate so that the function of the product could be negatively affected.

Such issues are commonly avoided by specifying tighter tolerances on part features. This requires that the parts are manufactured with more accurate processes, which are usually slower and more expensive. To get a good compromise between quality and cost, the designer should find out which tolerances have the most influence on the variation of the assembly dimension, and reduce them more than others with less influence. These decisions can be optimized by solving a problem of tolerance allocation: given the allowable variation

Antonio Armillotta

antonio.armillotta@polimi.it

1 Dipartimento di Meccanica, Politecnico di Milano, Via La Masa 1, 20156 Milan, Italy on the assembly dimension, determine the set of tolerances that minimizes the total manufacturing cost.

In its traditional formulation, tolerance allocation regards the tolerances as the only variables to be optimized. However, the error stackup depends on the nominal dimensions of the features as well. In principle, these could be optimized together with the tolerances, so that the specified assembly variation could be met with wider tolerances on the individual parts.

The optimization of nominal dimensions is a special case of robust parameter design, a problem aimed to reducing output variation in any type of system. In literature, the problem has been tackled using two main approaches. The first one is based on experimental or simulation plans, which are suitable for complex systems that cannot be described by simple mathematical models. The second one is based on analytical or numerical optimization, which allows an easier search for solutions in continuous spaces of many variables, with possible constraints and multiple objectives. Robust optimization seems to have more applications in tolerancing, probably due to the availability of mathematical methods for stackup analysis. In few cases, however, dimensions and tolerances have been treated jointly in the same optimization problem.

Integrating dimension and tolerance optimization is the main goal of this paper. Although in principle the robust 
allocation problem would make sense for any type of assembly, the study will focus on planar linkages for applications in structures and mechanisms. These are interesting for tolerancing problems as their complex layout may involve some difficulties in stackup calculations; moreover, they lend themselves to a simple, standard definition of the optimization problem. The proposed method treats the dimensions as direct optimization variables, while the tolerances are set in background using conditions for optimal allocation. These are found using an analytical optimization method (Lagrange multipliers), a cost-tolerance function including the effect of dimensions, and a method for stackup analysis based on a static analogy.

The paper is structured as follows. Section 2 reviews available studies on parameter and tolerance design for generic systems, mechanical assemblies and linkages. Section 3 defines the optimization problem and the assumptions underlying the proposed method, which is described in Section 4 and demonstrated on two examples in Section 5. The conclusions in Section 6 discuss the benefits and limitations of the method.

\section{Background}

The work is related to several research topics as discussed below.

\subsection{Robust parameter design}

A system is designed with the goal of improving its outputs through an optimal setting of its inputs. However, this may not guarantee a consistent behavior of the system because of variation sources (noise factors and errors in the input values), which may cause excessive output variation. Taguchi introduced the concept of robust design, which aims to reduce the effect of variation sources on outputs [1,2]. He also proposed methods based on design of experiments (DOE) to achieve this goal $[3,4]$. The core of Taguchi methods is parameter design, which uses an experimental plan based on orthogonal arrays to select the settings of system inputs (control factors) that are less sensitive to noise factors. Applications of the methodology to the design of manufactured products are reported in [5-7]. Attempts were also made to translate the Taguchi concepts into either practical design principles [8], or metrics to evaluate the robustness of a design [9].

Alternative DOE approaches to parameter design are mostly based on the response surface methodology (RSM). Its advantages on Taguchi methods include more efficient plans and the estimation of interaction effects between control factors, which can drive the search for optimal solutions [10-13]. Extensions of Taguchi and RSM approaches have been proposed to treat cases with multiple outputs or multi-objective decisions [14-16], and to improve the estimation of interaction effects [17]. Other studies adopt different experimental strategies and criteria for parameter selection $[18,19]$.

Robust optimization methods have been proposed as an alternative to DOE when dealing with complex cases involving many variables, constraints, unknown output functions, or statistical distributions [20-23]. The selection of parameter settings is modeled as an optimization problem in a continuous domain; the proposed objective functions include the output range for a given input setting [24-26], and the effect of the uncertainty in the model used for output calculation [27].

\subsection{Tolerance allocation}

The problem of tolerance allocation for a mechanical assembly has been studied extensively in literature. Given the allowable variation on the output (the assembly dimension), the goal is to determine the tolerances on the part dimensions that are linked to the output through a chain of assembly relationships (tolerance chain). This is described by an exact or approximate stackup function, which can be found by either geometric inspection or one of the available methods of tolerance analysis [28]. The optimal allocation is usually the one that minimizes a cost function while satisfying the constraint on output variation.

Introductions to tolerance allocation and classic application examples are given in $[29,30]$. A recent review on allocation methods [31] cites about 250 studies, classifying them in a detailed taxonomy. This includes the definition of the optimization problem (objective functions, constraints), the costtolerance models, and the assumptions on assembly requirements, tolerances (dimensional or geometric), and stackup criteria (worst-case, statistical). Other reviews [32,33] are also recommended for a full account of available approaches.

Most methods use search algorithms to find optimal tolerance allocations. In this work, an analytical solution is preferred for an easier integration with parameter optimization. In literature, analytical methods have been proposed under simplified yet realistic assumptions (dimensional errors with normal distributions, linear or first-order Taylor stackup functions). The most widely known is the method of Lagrange multipliers [34], which is also the basis for many numerical solutions.

Robust design concepts have been applied to tolerance allocation, in the attempt to balance the conflicting criteria of reducing cost and output variation. As in generic design problems, DOE and optimization are the two main routes to robust allocation.

In Taguchi methods, tolerance design is carried out after parameter design [35]. It is based on the quality loss function, which expresses the economic impact of deviations from the target output value. The concept is used to allocate tolerances on the control factors of the system, once an experiment has allowed to estimate the contribution of each factor to the 
overall quality loss. Modifications of Taguchi's tolerance design have been proposed in [36-38]. Other DOE approaches replace the quality loss with alternative selection criteria including the output variance [39] and various formulations of a cost function [40-43].

In optimization methods, quality measures are introduced into the objective functions or the constraints of the problem. Two common choices for the quality measure are the quality loss and the nonconforming rate. In the first approach (design for quality), the objective function is a sum of the manufacturing cost and the quality loss; this defines an unconstrained optimization problem, which has been solved with several search algorithms (see again [31-33] for reviews) and applied to some special formulations of the tolerancing problem [44-52]. In the second approach (design for reliability), several formulations have been proposed: minimize cost with a constraint on the nonconforming rate [53-57], maximize a process capability index [58], or optimize a function of cost and various indices related to the nonconforming rate [59-61]. Other formulations and choices of the objective function are discussed in [62-66].

\subsection{Optimal selection of dimensions and tolerances}

A few studies have dealt with the optimization of both dimensions and tolerances. This usually involves extending tolerance allocation problems by adding one or more dimensions as optimization variables, with constraints on the allowable design ranges. The sum of cost and quality loss (or a function of nonconforming rate) is minimized with several methods in problems involving single dimensions [67-69] or tolerance chains [70-74]. In [75], the cost is evaluated as the sum of separate elements depending on parameters and tolerances, while the output range is treated as a constraint. In [76, 77], a multi-objective genetic algorithm is used to optimize regression functions of cost. In [78], a Pareto frontier is generated to balance cost optimization with application-dependent performance metrics. In the RSM-based method of [79], a modified process capability index is defined to balance the conflicting objectives of minimum cost and maximum process capability.

An additional requirement with respect to plain tolerance allocation is that the solution space must also satisfy the geometric closure of the assembly as well as possible structural or functional requirements. These issues are usually overcome by solving two sequential optimization problems. In [80], the dimensions are found by minimizing the output variance for a tentative tolerance setting; then, the tolerances are allocated by minimizing a linear combination of cost and output variance. In [81], the dimensions are selected by minimizing quality loss using RSM on a plan of simulations; then, the tolerances are refined by minimizing an objective function including quality loss and cost of scrap or rework. In the single-variable problem of [82], the two stages optimize process means and variances with respect to cost and quality loss by closed formulas.

In the attempt to avoid two separate optimization stages, [83] minimizes the total cost with a constraint on the assembly dimension by Lagrange multipliers with numerical solution; unlike other cases, the optimal dimensions are selected in narrow ranges around reference values satisfying geometric and functional constraints. Other methods join the two stages by excluding cost from the objective function. [84] minimizes the variance of an overall performance measure given by the weighted sum of the variances of the functional requirements. In [85], process means and tolerances are selected with a geometric procedure that finds the points furthest from the boundaries of the allowable ranges in the space of nominal dimensions.

\subsection{Tolerance allocation on linkages}

The above problems have also been studied for some types of linkages. The design of parameters is usually done by extending classical design problems in order to comply with specific structural or kinematic requirements. For structures, robust design optimization is an extension of deterministic structural optimization with random uncertainty sources including dimensional errors [86-89]. For mechanisms, the kinematic synthesis problem has been extended to a stochastic formulation to consider the effects of dimensional errors on the desired kinematic function [90-96].

Tolerance allocation on mechanisms is often addressed by minimizing output variation over a set of different poses; the output is usually defined as the position of a point of interest. In [97], Lagrange multipliers are used in two optimization stages, which optimize output variation and cost. In [98], Lagrange multipliers are combined with a penalty function to minimize the cost with stackup constraints. In [99, 100], the output variation is minimized with a cost constraint. Taguchi-like methods have been proposed with different experimental designs to take into account kinematic and dynamic parameters [101, 102], joint clearances and additional errors from assembly and operating conditions [103], and sets of poses of the mechanism with reduction to a single performance index [104]. Both experimental and optimization methods have been attempted for specific types of mechanisms such as robotic manipulators $[105,106]$. A related problem has also been addressed for machine tools through the statistical analysis of multibody models [107].

In the above studies, dimensions and tolerances are usually optimized in distinct phases of mechanism design, namely kinematic synthesis (with given tolerances) and tolerance allocation (with given dimensions). In one case, however, the two phases are joined into a single problem. It consists in a robust optimization based on a graphical model, an ellipsoid in the space of variables, whose semi-axes are in inverse 
relationship with the sensitivity of the output to the corresponding variables [108]. This allows to express the objective of robust design by geometric conditions on the ellipsoid (size, aspect ratio, and orientation); the conformance is checked by comparing the ellipsoid with a tolerance box representing the tolerances specified on the variables [109].

\subsection{Overview}

The hypothesis of this work is that the dimensional parameters of an assembly can be optimized together with the tolerances in order to reduce the output variation due to manufacturing errors. It is apparent that this objective has been recognized in a diversity of design problems well beyond the field of tolerancing. Robust parameter design as a DOE methodology allows to treat complex systems whose outputs can only be evaluated by physical testing or computer simulation. However, it is not suitable to problems with many variables, constraints, and interaction effects; the parameters are selected from discrete values, and optimal settings can only be found by iterations of the experiments. These limits are overcome by robust optimization methods, which require continuous and possibly differentiable output functions. In both approaches, parameters and tolerances are usually selected in distinct phases rather than in a single optimization problem.

The same dichotomy between DOE-based and optimization approaches applies to tolerance allocation. The availability of methods for tolerance stackup calculations justifies a relative preference for optimization methods, which incorporate robustness by adding the quality loss or the nonconforming rate to either the objective functions or the constraints. However, the allocation problem is usually defined with given dimensions. The separate optimization of dimensions and tolerances has been attempted with DOE approaches or multi-objective problems, where the two usual objectives (output variance and cost) are combined with weighting functions or kept separate for a final selection of Pareto-optimal solutions. The effective integration of the two problems seems to be restricted to special formulations, which include single-dimension problems, selection of parameters in narrow ranges, or optimization problems without cost functions.

Tolerance allocation on linkages has been mostly studied for applications on path-generating mechanisms, where the optimization spans a set of positions of the tracing point. The robust optimization of dimensions and tolerances has been perceived as a useful extension of the allocation problem with given dimensions, with promising theoretical contributions (such as the sensitivity ellipsoid) that could pave the way for the treatment of increasingly complex design cases.

The literature review seems to justify the goal of a seamless optimization of dimensions and tolerances. An aspect that has been relatively neglected, and which will be discussed below, is that treating dimensions as additional optimization variables emphasizes the need of cost models including the influence of dimensions as well as tolerances. Such a model has been proposed in a recent study [110] as an extended formulation of one of the available cost-tolerance functions, and will be used in this study.

\section{Problem and assumptions}

This work will consider planar linkages with rigid links connected by revolute and prismatic joints. They are assumed to be exactly constrained; this condition applies to isostatic structures such as trusses, but also to mechanisms with a degree of freedom locked in a reference pose. These assumptions cover a wide diversity of function-generating and path-generating mechanisms; the proposed method does not place limits on the number of links and joints, although particularly complex mechanisms may require the analysis of many poses as well as a greater amount of calculations for each pose. The extension to mechanisms with higher kinematic pairs may require further developments for the necessary extensions of the calculation procedures.

The output $Y$ defined on the linkage is a linear or angular dimension defined at assembly level, such as the absolute position of a point along a certain direction, the distance between two joints, or the orientation of a link. The output is to be controlled within a tolerance $T_{Y}$ from a nominal value $Y_{0}$ to ensure the desired function of the linkage.

The output depends on $n$ dimensions $X_{i}$ of individual parts; they include the lengths of links, and the diameters of holes and pins. The relationship between the output and the dimensions is assumed to be a linear (or linearized) stackup equation:

$Y=\sum_{i=1}^{n} S_{i} X_{i}$

where $S_{i}$ is the sensitivity of the output to dimension $X_{i}$.

Each dimension has a nominal value $X_{0 i}$ and a tolerance $T_{i}$, which can be controlled through the manufacturing process of the part. In statistical tolerancing, the dimensions $X_{\mathrm{i}}$ are assumed to be independent and normally distributed. Their means are equal to the $X_{0 i}$, and their standard deviations $\sigma_{i}$ are in a constant proportion with the $T_{i}$. The nonconforming rate is assumed to be low, e.g., $\leq 0.27 \%$ if $T_{i} \geq 3 \sigma_{i}$. Under these assumptions, $Y$ is also normally distributed with mean $Y_{0}$ and standard deviation in the same proportion with $T_{0}$; the output tolerance is calculated from part tolerances through the root-sum-square (RSS) equation:

$T_{Y}=\sqrt{\sum_{i=1}^{n} S_{i}^{2} T_{i}^{2}}$ 
If any of the above assumptions is violated, the stackup of the input tolerances results in a higher output error due to various reasons (covariance terms, linear accumulation of systematic errors, etc.). These effects are accounted for by a correction factor $c \geq 1$ in the RSS equation [29]:

$T_{Y}=c \sqrt{\sum_{i=1}^{n} S_{i}^{2} T_{i}^{2}}$

A 50\% increase of the output tolerance is often assumed $(c=1.5)$, although more accurate statistical expressions of the correction factor are available [111].

In tolerance allocation, the $X_{0 i}$ are given and the $T_{i}$ are the variables of the following optimization problem:

$$
\begin{aligned}
& \min \sum_{i=1}^{n} f_{i}\left(T_{i}\right) \\
& \text { s.t. } T_{Y}^{2}=c^{2} \sum_{i=1}^{n} S_{i}^{2} T_{i}^{2}
\end{aligned}
$$

where $f_{i}\left(T_{i}\right)$ is the cost-tolerance function for $X_{i}$. Further constraints on the values of the individual $T_{i}$ can be avoided assuming that the minimum cost is always reached within the allowable tolerance ranges.

The proposed extension considers the $X_{0 i}$ as optimization variables in addition to the $T_{i}$. That is, in addition to the optimal tolerances, the optimal values of the nominal dimensions are also searched for in the domain of possible configurations of the linkage. Just like the tolerances, the dimensions must also meet some conditions, which include the following:

- Constant values possibly set for some dimensions in order to control the overall size of the linkage;

- The nominal value of the output dimension:

$$
Y_{0}=\sum_{i=1}^{n} S_{i} X_{0 i}
$$

- Relationships that must occur between the dimensions in order to ensure the geometric closure of the assembly; as a simple example, in a rectangular truss the lengths of two links (one horizontal and one vertical side) determine the lengths of all the other links (the opposite sides and the diagonal).

The optimization problem could be formally defined by adding constraints for these conditions to (1). However, this choice would lead to different constraint equations for each different case, and would lose the chance of a solution in common form for all possible linkages. For the purposes of this work, it is rather suggested that the designer identifies a set of independent dimensions, i.e., those that can be freely optimized without violating the above conditions. These will be treated as the optimization variables, while the other nonconstant dimensions will be expressed as functions of the independent ones.

The independent dimensions may even be chosen outside the set of $X_{0 i}$; in some cases, it might be better to optimize auxiliary geometric variables, such as an angle (e.g., to determine two sides of a right triangle) or a ratio between two dimensions (to allow their choice in a wide range while controlling the aspect ratio of the linkage). Any possible set of independent dimensions is likely to lead to the same optimal set of $X_{0 i}$; the choice should generally be guided by the need for simplicity and clarity of the geometric relationships. When dealing with mechanisms, however, explicit constraints may need to be added to the optimization problem in order to avoid solutions that are technically not feasible, e.g., causing the jamming of a mechanism. In this paper, such possibility will not be considered, and any kinematic and static verification of the linkage will be assumed to have been carried out according to the type and function of the mechanism being designed. General criteria for these tasks are currently dealt with in kinematic synthesis, where the parameters of a mechanism are optimized either deterministically or including the effects of random errors with given tolerances [90-96].

The above defined optimization problem has two more difficulties than plain tolerance allocation:

- The cost-tolerance functions depend on the nominal dimensions: a given tolerance is more expensive to satisfy on a larger dimension due to some variation sources in the manufacturing process (e.g., deformations of workpieces and fixtures);

- The sensitivities $S_{i}$ depend on the nominal dimensions: these determine the layout of the linkage, and therefore the geometric relationships among the different links and joints.

\section{Method}

The proposed solution is based on a known method for tolerance allocation, which is used to establish analytical conditions on the optimal tolerances. These are expressed as a function of the dimensions using a suitable cost-tolerance function. An optimization problem is then defined for a set of independent dimensions. Its solution requires the calculation of sensitivities, which is done through a tolerance analysis method based on a static analogy.

\subsection{Optimal allocation}

For any choice of dimensions, the optimal tolerances are the solution of the allocation problem (1). The Lagrange 
multiplier method [34] finds them by solving the following unconstrained optimization problem:

$\min C=\sum_{i=1}^{n} f_{i}\left(T_{i}\right)+\lambda\left(T_{Y}^{2}-c^{2} \sum_{i=1}^{n} S_{i}^{2} T_{i}^{2}\right)$

The multiplier $\lambda$ is calculated by equating the derivatives $\partial C / \partial T_{i}$ to zero:

$\lambda=\frac{\partial f_{i} / \partial T_{i}}{2 c^{2} S_{i}^{2} T_{i}}$

The above expression can be made explicit by choosing a relatively simple form for $f_{i}\left(T_{i}\right)$, such as the reciprocal power function:

$f_{i}=a_{i}+\frac{b_{i}}{T_{i}^{k}}$

where $a_{i}$ is a fixed cost with respect to tolerance, while $b_{i}$ and $k$ are the factor and the exponent of the variable cost with respect to tolerance (the only parameters that appear in $\partial C / \partial T_{i}$ ). As $\lambda$ is constant for $i=1, \ldots n$, the following analytical relationship among the optimal tolerances is found:

$\left(T_{i}\right)_{\mathrm{opt}} \propto\left(\frac{b_{i}}{S_{i}^{2}}\right)^{\frac{1}{k+2}}$

These known results will be the starting point to build a method for the simultaneous optimization of dimensions and tolerances. The method of Lagrange multipliers with RSS equation and reciprocal power cost-tolerance function could seem a limiting choice: in fact the allocation problem is treated in literature with less restrictive assumptions on the distributions of dimensions (not normal), on the stackup functions (not linear, or even not known a priori), and on the very nature of the optimization problem (with discrete variables in the presence of alternative choices on processes and equipment). The proposed approach may be justified by the objective of verifying the feasibility of extending the problem considering the various types of constraints (geometric, functional, structural) that may occur on part dimensions. Based on existing approaches, it is not obvious that the improvements over classical tolerance allocation outweigh possible complications in defining and solving the problem. It is believed that the answer to these questions should be sought in the simplest formulation of tolerancing problems, where an assembly dimension is determined by the linearized stackup of part dimensions satisfying basic statistical assumptions (independence, normal distributions, limited nonconforming rate). This will allow a sufficiently simple analytical formulation, on which the possible benefits of the extension can be more easily evaluated.

\subsection{Cost-tolerance function}

As mentioned, the cost parameters in (2) depend on the dimensions. The reciprocal power function has been widely used in literature assuming values for $b_{i}$ and $k$ from application-dependent cost data. In an expanded form of the same function proposed in [110], the exponent is constant and the factor is empirically related to the nominal dimension:

$b_{i}=\beta_{i} X_{0 i}^{k / 3}$

Therefore, the variable cost of a feature depends on its nominal dimension through a power-law relationship with an exponent equal to one-third of that of its tolerance. This result was obtained in [110] through the regression of data from several sources on the costs of machining processes as a function of the tolerance grades on the machined features. The same analysis led to the estimate of a value $k=0.55$ for the exponent of the cost-tolerance function, which should be verified on real cost data in specific production contexts.

The coefficient $\beta_{i}$ depends on the properties of the part feature associated to the dimension:

$\beta_{i}=f_{\mathrm{A} i} \cdot f_{\mathrm{F} i} \cdot f_{\mathrm{M} i} \cdot b_{0}$

where:

- $f_{\mathrm{A} i}$ is the area of the feature in $\mathrm{cm}^{2}$;

- $f_{\mathrm{F} i}$ depends on the shape of the feature; possible values on rotational parts are 0.75 and 1.25 respectively for external and internal features; on prismatic parts, 1.5 for cylindrical features, 1-1.5 for non-cylindrical features, and 0.5-1 for planar features (higher values for smaller features);

- $f_{\mathrm{M} i}$ is inversely related to the machinability of the material, with base 1 for mild steel, in a range from about 0.3 for aluminum alloys to about 2 for stainless steels;

- $b_{0}$ is a constant that does not influence the allocation results $\left(b_{0}=1.0 \cdot 10^{-3}\right.$ from [110]).

Compared to the basic reciprocal power function, the expanded form makes explicit the influence of the nominal dimension in the cost of a feature. As discussed in [110], this should improve the consistency of the results of tolerance allocation, as the costs of all features involved in a tolerance chain are more easily estimated on a common scale. The function is even more useful in the present work, where the nominal dimensions are treated as additional variables of the optimization problem.

Equation (4) was obtained for linear dimensions, but can be extended to angular dimensions. However, the cost of an angular dimension is likely to depend not so much on the nominal angle as on the adjacent linear dimensions. In a general situation of the type illustrated in Fig. 1, an angle $\theta$ in radians is limited by two linear features (e.g., edges of a part) with 


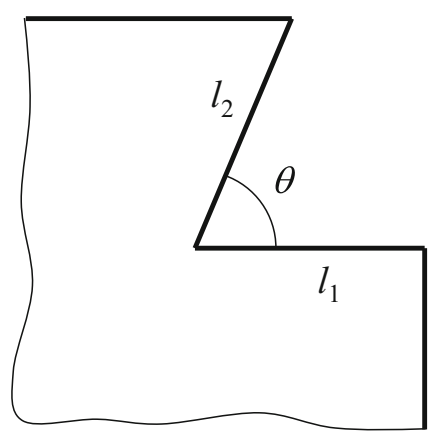

Fig. 1 Tolerance on an angular dimension

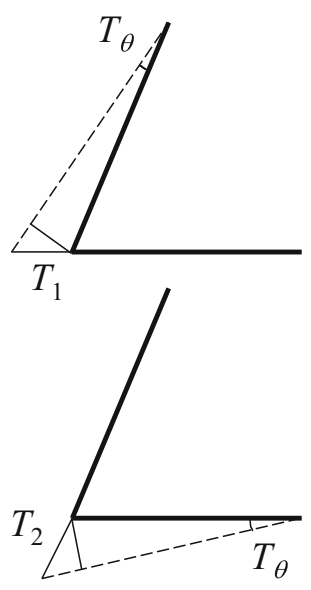

lengths $l_{1}$ and $l_{2}$. If one of the two elements is subject to tolerance $\left(T_{1}\right.$ or $\left.T_{2}\right)$, the resulting variation on $\theta$ is

$T_{\theta}=\frac{T_{1} \sin \theta}{l_{2}}$ or $T_{\theta}=\frac{T_{2} \sin \theta}{l_{1}}$

For simplicity, it can be assumed that $T_{\theta}$ is related to the geometric mean $l$ of the two lengths:

$T_{\theta}=\frac{T_{l} \sin \theta}{l}, l=\sqrt{l_{1} l_{2}}$

In (4), the angle must be associated to an equivalent nominal dimension $X_{\theta}$, which has the same cost as $l$ for the same variation. Considering only the variable cost, (2) and (4) give the condition

$\frac{X_{\theta}^{k / 3}}{T_{\theta}^{k}}=\frac{l^{k / 3}}{T_{l}^{k}}$

which combined with (6) becomes

$\frac{X_{\theta}^{k / 3}}{T_{\theta}^{k}}=\frac{\sin ^{k} \theta}{T_{\theta}^{k} l^{2 k / 3}} \rightarrow X_{\theta}=\frac{\sin ^{3} \theta}{l^{2}}=\frac{\sin ^{3} \theta}{l_{1} l_{2}}$

Therefore, the expanded form (4) of the variable cost factor can also be used for an angular dimension in radians by replacing the nominal angle with an appropriate function of the angle and of the adjacent linear dimensions.

Substituting (4) into (3) gives a new condition on optimal tolerances, which explicitly takes into account the nominal dimension and the properties of the feature:

$\left(T_{i}\right)_{\mathrm{opt}} \propto\left(\frac{\beta_{i} X_{0 i}^{k / 3}}{S_{i}^{2}}\right)^{\frac{1}{k+2}}$

Without loss of generality, it is now assumed that all the links are similar for material, shape, section size, and hole diameters. This implies that the coefficients $\beta_{i}$ have the same values for all the $X_{i}$. This can be justified by considering the product $\left(f_{\mathrm{A} i} \cdot f_{\mathrm{F} i}\right)$ that appears in the expression (5) of $\beta_{i}$. The features are either the holes (for the link lengths and the hole diameters) or the cylindrical surfaces of the pins (for the pin diameters). The pin surfaces have double area $f_{\mathrm{A} i}$ with respect to the holes (assuming that each pin connects two links), but a shape coefficient $f_{\mathrm{F} i}$ half that of the holes ( 0.75 vs 1.5$)$, and thus equal value of their product. As a result, the condition (8) on optimal tolerances for the linkage simplifies to

$$
\left(T_{i}\right)_{\mathrm{opt}} \propto\left(\frac{X_{0 i}^{k / 3}}{S_{i}^{2}}\right)^{\frac{1}{k+2}}
$$

\subsection{Objective function for nominal dimensions}

The optimal dimensions can be found by solving another optimization problem whose only variables are the $X_{0 i}$. The objective function is the stackup of the optimal tolerances

$\sigma=c \sqrt{\sum_{i=1}^{n} S_{i}^{2} T_{i}^{2}}$

where the $T_{i}$ satisfy the condition (9) for each possible set of $X_{i}$. To optimize the output variance $\sigma^{2}$, the problem can be defined as

$\min \sum_{i=1}^{n} S_{i}^{2}\left(\frac{X_{0 i}^{k / 3}}{S_{i}^{2}}\right)^{\frac{2}{k+2}}=\sum_{i=1}^{n} X_{0 i}^{a}\left|S_{i}\right|^{3 a}, \quad a=\frac{2 k}{3(k+2)}(10)$

with $a=0.14$ for $k=0.55$. The overall problem is therefore solved concurrently by two nested optimizations: minimumvariance dimensions are found by assuming minimum-cost conditions for the tolerances. Once the optimal dimensions are known, the optimal tolerances are scaled to the specified output tolerance $T_{Y}$ :

$T_{i}=\frac{T_{Y}}{c} \frac{\left(T_{i} / T_{1}\right)}{\sqrt{\sum_{i=1}^{n} S_{i}^{2}\left(T_{i} / T_{1}\right)^{2}}}, \quad \frac{T_{i}}{T_{1}}=\left(\frac{X_{0 i}^{k / 3}}{X_{01}^{k / 3}} \frac{S_{1}^{2}}{S_{i}^{2}}\right)^{\frac{1}{k+2}}$

This last step should not be considered a second optimization phase, as the proportions between the tolerances are already included in condition (9) and therefore incorporated by (10). The underlying assumption is that the optimality condition does not depend on the tolerance specification on the output dimension. This is not guaranteed, however, because cost-tolerance functions are not linear; a proportional increase of all tolerances would thus change the relationships between the corresponding costs, which may yield a total cost that is no longer optimal. To verify if the nested optimizations are able 
to approximate the solution of the original optimization problem (1), the solution space must be explored in the search of the minimum cost over the acceptable combinations of independent dimensions. In the following, such a verification will be made on two simple examples by enumerative search; in more complex cases, different constrained or unconstrained search algorithms may be required.

\subsection{Calculation of sensitivities}

In the objective function (10), the sensitivities $S_{i}=\partial Y / \partial X_{i}$ depend on the dimensions. Given the layout of the linkage, the $S_{i}$ can be calculated with one of the tolerance analysis methods available in literature. The method that will be used here was previously proposed for rigid and exactly constrained assemblies [112] and later demonstrated on planar linkages [113].

The method is based on a static analogy, which converts the tolerance analysis problem into an equivalent problem of force analysis. In the linkage of Fig. 2a, the output $Y$ is the position of a point along a given direction and has unknown sensitivities to the dimensions of the parts (lengths of links, diameters of holes and pins). As shown in Fig. $2 b$, the problem is solved by considering an equivalent static model, where an external force $F$ is applied to the output point along the output direction. The analysis of the forces on the static model requires a free-body diagram to show the support reactions and the forces between the links; these are calculated from the equilibrium equations, which are equal in number to the unknown force components as the linkage is exactly constrained. The solution of the static model gives the axial internal forces $F_{i}$ in the

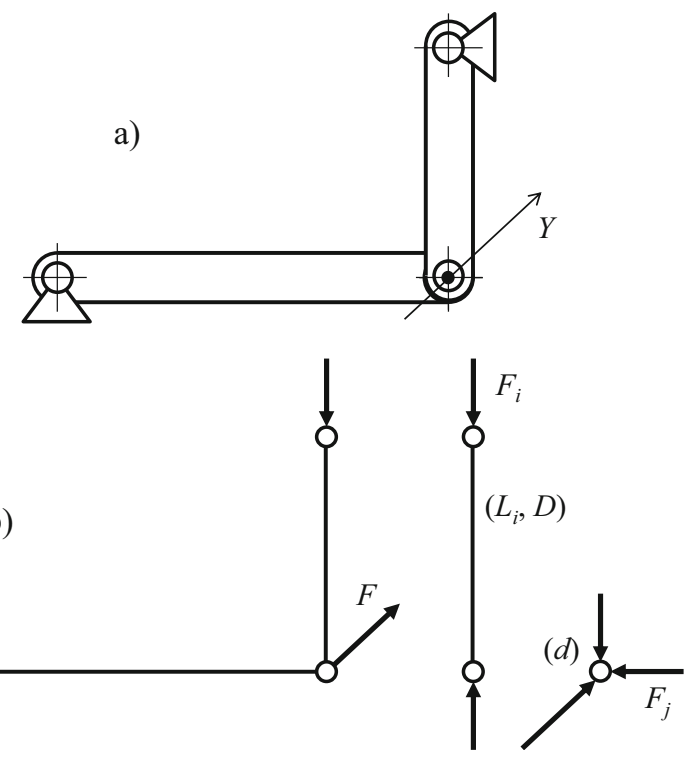

Fig. 2 Static analogy: (a) output and dimensions; (b) equivalent static model links. The sensitivities of the output to link lengths are then found dividing the internal forces by the external force:

$S_{i}=\frac{F_{i}}{F}$

Other types of dimensions and outputs can be treated similarly. If the output is the distance between two points of the linkage, opposite forces are applied to the two points in the direction of their distance. For angular dimensions or outputs, the internal or external force is replaced by a torque. A mechanism can be analyzed in one of its poses by locking its degrees of freedom with additional constraints; the internal force or torque corresponding to each constraint gives the sensitivity of the locked linear or angular displacement.

Due to joint clearances, the output also depends on diameters $D$ and $d$ of holes and pins. Their sensitivities are calculated as

$S_{D}=\frac{1}{2} \frac{F_{i}}{F}$

$S_{d}=-\frac{1}{2} \sum_{j} \frac{F_{j}}{F}$

where $F_{i}$ is the axial force of the same link of a hole and the $F_{j}$ are the radial forces on a pin by adjacent links or supports (Fig. 2b).

As discussed in [113], the static analogy derives from the application of the principle of virtual work to the equilibrium condition on an assembly or linkage. Although it has only been demonstrated on planar systems, the analogy could therefore be extended to three-dimensional problems. This would require further developments to deal with new cases of geometric errors (e.g., angular errors in different directions), and to establish their correspondences with new types of internal forces (e.g., twisting moments or bending moments in different directions). In the treatment of spatial mechanisms, further types of joints would have to be considered (e.g., helical or spherical joints); moreover, it is likely that the solution of some 3D static models is so complex to require a dedicated software implementation.

\section{Examples}

The method will now be demonstrated on two examples: a structure with one independent dimension, and a mechanism with two independent dimensions.

\subsection{Structure}

Figure 3 shows a truss with three members, simply supported at points $\mathrm{A}$ and $\mathrm{B}$. The upper member has nominal length $L_{1}$ 


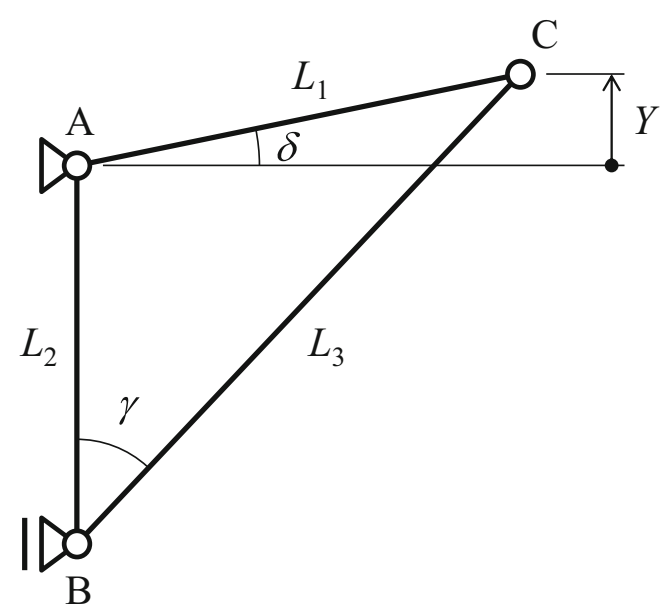

Fig. 3 Truss structure

and nominal angle $\delta$ to the horizontal. All the joints include pins with nominal diameter $d$ and holes with nominal diameter $D=d$, connected with a line fit. The optimization variables include the nominal dimensions $L_{2}$ and $L_{3}$, and the tolerances on all dimensions $\left(L_{1}, L_{2}, L_{3}, D, d\right)$. The output is the vertical position $Y$ of point $\mathrm{C}$ with respect to A (nominally equal to $L_{1}$ $\sin \delta$ ), for which a tolerance $T_{Y}$ is specified.

From Eqs. (1), (2), and (4), the cost associated to dimension $i$ can be expressed as

$f_{i}\left(T_{i}\right)=a_{i}+\frac{\beta_{i} X_{0 i}^{k / 3}}{T_{i}^{k}}$

The cost function can be simplified by excluding the fixed cost $a_{i}$ and considering that, under the assumptions discussed in the comment to Eq. (8), the coefficient $\beta_{i}$ is equal for all the dimensions of a planar linkage. A suitable objective function $f_{C}$ is thus the sum of the variable costs divided by the common coefficient $\beta$. From (15), such function has the following expression:

$f_{C}=\sum_{i=1}^{n} \frac{X_{0 i}^{k / 3}}{T_{i}^{k}}$

Therefore, the optimization problem (1) can be stated as follows:

$\min f_{C}=\frac{L_{1}^{k / 3}}{T_{1}^{k}}+\frac{L_{2}^{k / 3}}{T_{2}^{k}}+\frac{L_{3}^{k / 3}}{T_{3}^{k}}+\frac{D^{k / 3}}{T_{D}^{k}}+\frac{d^{k / 3}}{T_{d}^{k}}$

s.t. $T_{Y}^{2}=c^{2}\left(S_{1}^{2} T_{1}^{2}+S_{2}^{2} T_{2}^{2}+S_{3}^{2} T_{3}^{2}+S_{4}^{2} T_{D}^{2}+S_{5}^{2} T_{d}^{2}\right)$

As discussed in subsection 4.2, the solution to (17) will be found by solving the equivalent problem (10), where the sensitivities $S_{i}$ have now to be evaluated.

As the unknown dimensions $L_{2}$ and $L_{3}$ are dependent on each other, the angle $\gamma$ between the two members is taken as the optimization variable, so that
$L_{2}=\frac{\cos (\delta+\gamma)}{\sin \gamma} L_{1}, \quad L_{3}=\frac{\cos \delta}{\sin \gamma} L_{1}$

The equivalent static model is defined as shown in Fig. 4a: a vertical force $F$ is applied to $\mathrm{C}$ and causes support reactions in $\mathrm{A}$ and $\mathrm{B}$ for the equilibrium of the structure. The analysis of the free-body diagram in Fig. $4 \mathrm{~b}$ gives the forces acting on the links and pins.

The sensitivities of $Y$ to the lengths $X_{1}, X_{2}$, and $X_{3}$ of the links are calculated from (12):

$$
\begin{aligned}
& S_{1}=-\frac{L_{1}}{L_{2}} \\
& S_{2}=-1-\frac{L_{1}}{L_{2}} \sin \delta \\
& S_{3}=\frac{L_{1}}{L_{2}} \frac{\cos \delta}{\sin \gamma}
\end{aligned}
$$

For the diameters $D_{1}, D_{2}$, and $D_{3}$ of the holes, the sensitivities are calculated from (13):

$$
\begin{aligned}
S_{D 1} & =\frac{1}{2} \frac{L_{1}}{L_{2}} \\
S_{D 2} & =\frac{1}{2}\left(1+\frac{L_{1}}{L_{2}} \sin \delta\right) \\
S_{D 3} & =\frac{1}{2} \frac{L_{1}}{L_{2}} \frac{\cos \delta}{\sin \gamma}
\end{aligned}
$$

Similarly, the sensitivities related to the pin diameters $d_{\mathrm{A}}$, $d_{\mathrm{B}}$, and $d_{\mathrm{C}}$ are calculated from (14):

$$
\begin{aligned}
& S_{d \mathrm{~A}}=-\frac{1}{2}\left[1+\frac{L_{1}}{L_{2}}+1+\frac{L_{1}}{L_{2}} \sin \delta+\sqrt{1+\left(\frac{L_{1}}{L_{2}} \cos \delta\right)^{2}}\right] \\
& S_{d \mathrm{~B}}=-\frac{1}{2}\left(\frac{L_{1}}{L_{2}} \cos \delta+1+\frac{L_{1}}{L_{2}} \sin \delta+\frac{L_{1}}{L_{2}} \frac{\cos \delta}{\sin \gamma}\right) \\
& S_{d \mathrm{C}}=-\frac{1}{2}\left(1+\frac{L_{1}}{L_{2}}+\frac{L_{1}}{L_{2}} \frac{\cos \delta}{\sin \gamma}\right)
\end{aligned}
$$

Although the tolerances on individual diameters could be optimized separately, a common specification is assumed for all the holes $\left(X_{4}\right.$, with nominal diameter $\left.D\right)$ and for all the pins $\left(X_{5}\right.$, with nominal diameter $d$ ). Considering that there are two equal holes on each link, the sensitivities of $Y$ to these two variables are obtained as a function of $\gamma$ from the following equations:

$$
\begin{aligned}
& S_{4}=\sqrt{2 S_{D 1}^{2}+2 S_{D 2}^{2}+2 S_{D 3}^{2}} \\
& S_{5}=-\sqrt{S_{d \mathrm{~A}}^{2}+S_{d \mathrm{~B}}^{2}+S_{d \mathrm{C}}^{2}}
\end{aligned}
$$

From (10), the objective function for the dimensions is therefore

$\varphi(\gamma)=L_{1}^{a}\left|S_{1}\right|^{3 a}+L_{2}^{a}\left|S_{2}\right|^{3 a}+L_{3}^{a}\left|S_{3}\right|^{3 a}+D^{a}\left|S_{4}\right|^{3 a}+d^{a}\left|S_{5}\right|^{3 a}$ 
Fig. 4 Equivalent static model for the truss: (a) external force and support reactions; (b) free-body diagram

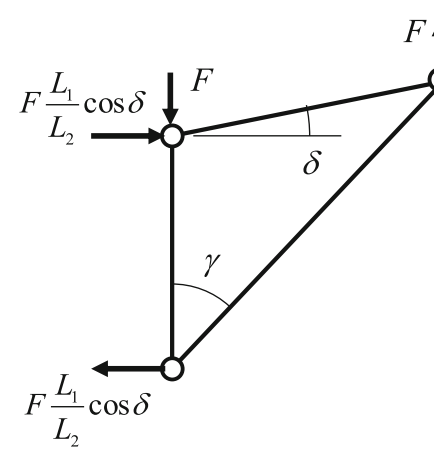

a)

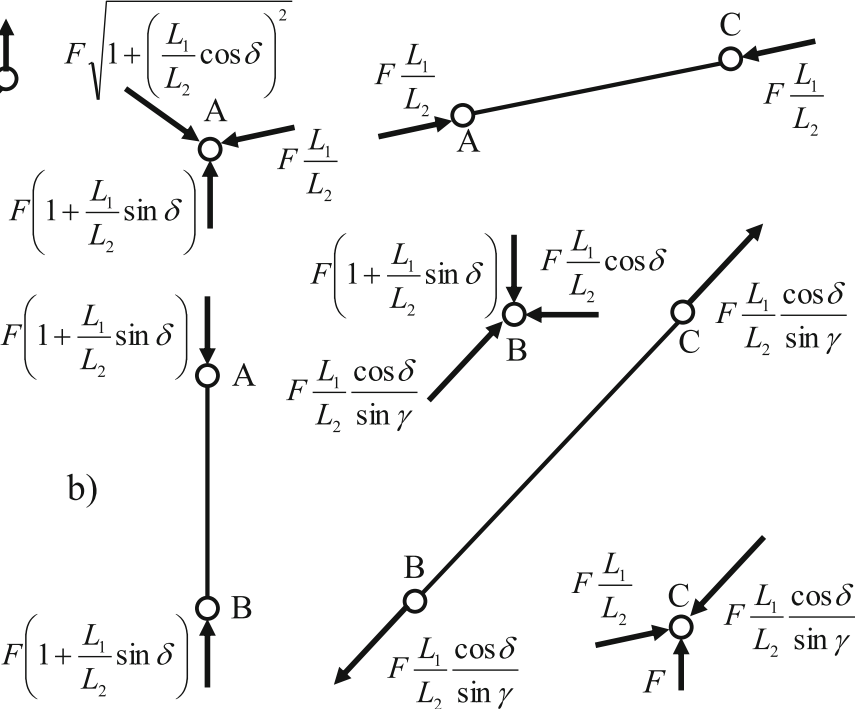

where $L_{1}, D$, and $d$ are constant, while $L_{2}, L_{3}$, and the sensitivities $S_{i}(i=1, \ldots 5)$ are functions of $\gamma$.

Figure 5 shows the graph of $\phi$ for $L_{1}=100 \mathrm{~mm}, D=d=$ $5 \mathrm{~mm}$, and $\delta=-30^{\circ}$; within the range $0^{\circ}<\gamma \leq 90^{\circ}$, the function has a minimum for $\gamma=32.9^{\circ}$, which determines the optimal dimensions $L_{2}=183.9 \mathrm{~mm}$ and $L_{3}=159.4 \mathrm{~mm}$. The figure also shows the graph of the function $\phi_{1}$ limited to the first three terms, which would have been used if the joint clearances were neglected. The difference between the two graphs suggests that such simplification would lead to underestimating the output variance by over $30 \%$, and to a different optimal solution $\left(\gamma=90^{\circ}\right)$.

The optimal tolerances $T_{i}(i=1, \ldots 5)$ can be calculated from (11), where $n=5$ and the nominal dimensions $X_{0 i}$ are $L_{1}, L_{2}, L_{3}, D$, and $d$. Assuming $T_{Y}=0.2 \mathrm{~mm}$ and $c=1.5$, this gives the optimal tolerances listed in Table 1 along with the

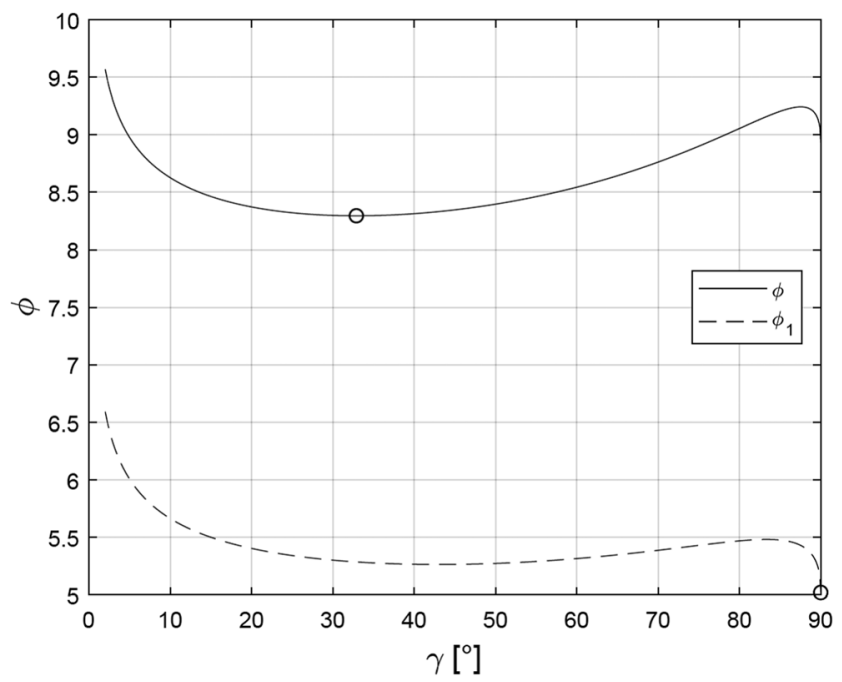

Fig. 5 Output variance for the truss nominal dimensions and sensitivities. Figure 6 shows a drawing of the optimized structure.

Figure 7 shows the graph of total cost $f_{C}$, the objective function in (17), obtained by finding the optimal tolerance allocation for the different configurations of the structure with the same values of $L_{1}, D, d$, and $\delta$. It is apparent that the variation of cost with the optimization variable follows a similar pattern to that of the output variance. The minimum cost differs by just $0.2 \%$ to that of the solution found with the proposed method (indicated on the graph), and corresponds to a very close value of the variable $\left(\gamma=27.4^{\circ}\right)$. When $\gamma$ increases beyond the optimal value, the cost increases significantly: in comparison to a solution with $\gamma=30^{\circ}$ (truss shaped as a right triangle in $\mathrm{C}$, very similar to the one illustrated in Fig. 6), the cost would increase by about $6 \%$ for $\gamma=60^{\circ}$ (truss shaped as an equilateral triangle), and by $25 \%$ for $\gamma=90^{\circ}$ (truss shaped as a right triangle in B).

\subsection{Mechanism}

Figure 8 shows a mechanism for the transmission of motion between two linear axes [114]. As the slider A moves along direction $\mathrm{OA}$, the tracing point $\mathrm{B}$ translates along direction

Table 1 Optimization results for the truss

\begin{tabular}{llll}
\hline Dimension & $X_{0 i}, \mathrm{~mm}$ & $T_{i}, \mathrm{~mm}$ & $\left|S_{i}\right|$ \\
\hline$X_{1}$ & $L_{1}=100$ & 0.104 & 0.544 \\
$X_{2}$ & $L_{2}=183.9$ & 0.086 & 0.728 \\
$X_{3}$ & $L_{3}=159.4$ & 0.075 & 0.867 \\
$X_{4}$ & $D=5$ & 0.057 & 0.888 \\
$X_{5}$ & $d=5$ & 0.027 & 2.318 \\
\hline
\end{tabular}




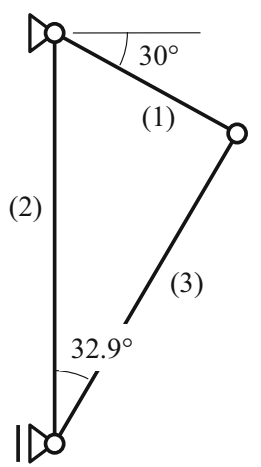

(1)

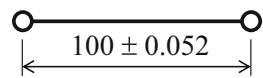

(2)

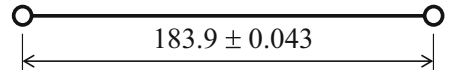

(3)

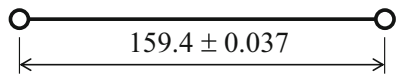

Fig. 6 Optimal dimensions and tolerances for the truss

$\mathrm{OB}$ at an angle $\delta$ with the perpendicular to OA. The lever AEC with dimensions $r_{A}$ and $h$ is connected to the lever CDB with dimensions $l$ and $r_{B}$; they have equal internal angles $\left(90^{\circ}-\delta\right)$ and proportional dimensions $\left(r_{A} / h=l / r_{B}\right)$. The followers OD and OE with lengths $h$ and $l$ are connected to levers AEC and $\mathrm{CDB}$, so as to form a parallelogram ODCE with an inside angle $\gamma$ in $\mathrm{O}$ and angles $\alpha$ and $\beta$ to the paths of $\mathrm{A}$ and $\mathrm{B}$, where

$$
\begin{aligned}
\tan \alpha & =\frac{r_{A} \cos (\gamma+\delta)}{l+r_{A} \sin (\gamma+\delta)} \\
\beta & =90^{\circ}-(\alpha+\gamma+\delta)
\end{aligned}
$$

As a result of the movements of the four links, the slider and the tracing point are both approaching or moving away from $\mathrm{O}$; their travel is limited by two extreme poses where the parallelogram is folded $(\gamma=0)$ or completely unfolded $(\gamma=$ $90^{\circ}-\delta$ ). The output $Y$ is the position of B along its path $\mathrm{OB}$ for a given position of $\mathrm{A}$, corresponding to $\gamma=45^{\circ}-\delta / 2$ (halfway from the extreme poses). A tolerance $T_{Y}$ must be satisfied at minimum cost by selecting both dimensions and tolerances. Given the nominal lengths of the outermost bars $\left(r_{A}=\right.$

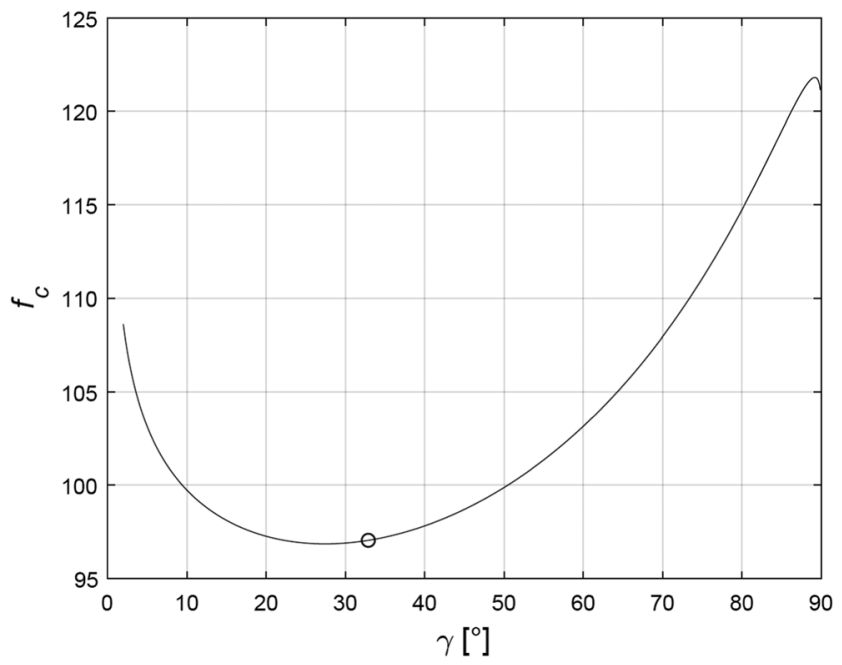

Fig. 7 Total cost for the truss

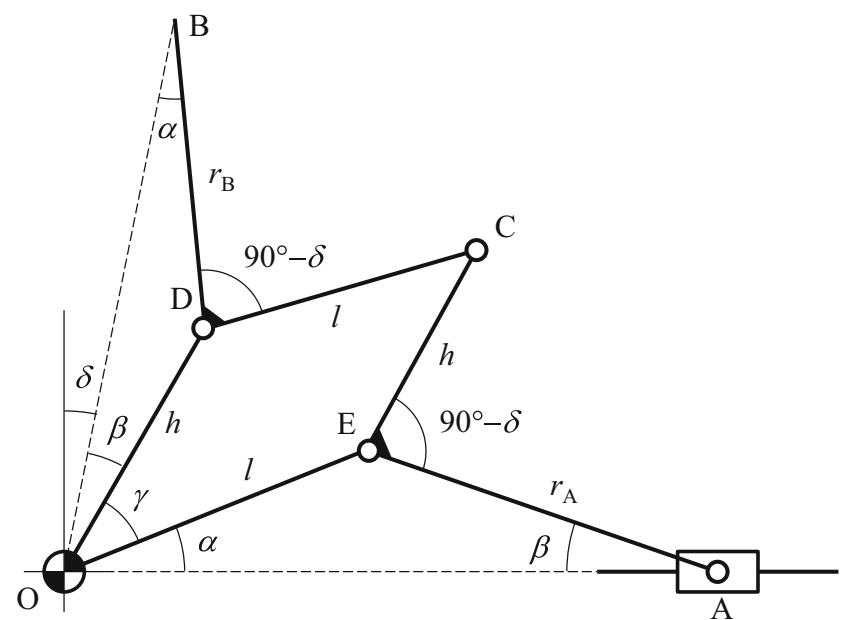

Fig. 8 Mechanism for transmission of linear displacement

$150 \mathrm{~mm}, r_{B}=200 \mathrm{~mm}$ ) and the nominal diameters of the joints ( $D=d=10 \mathrm{~mm}$ ), the problem boils down to two independent dimensions: angle $\delta$ and length $l$.

The dimensions influencing the output include six lengths $\left(X_{1}, \ldots X_{6}\right)$, two diameters $\left(X_{7}, X_{8}\right)$, and two angles $\left(X_{9}, X_{10}\right)$. Table 2 explains the meaning of the $X_{i}$ and lists the nominal dimensions $X_{0 i}$, some of which depend on the unknown independent dimensions $\delta$ and $l$. For the two angles, the nominal dimensions are set at equivalent values according to (7).

With the above notation, the optimization problem has the following definition:

$$
\begin{aligned}
\min f_{C} & =\sum_{i=1}^{10} \frac{X_{0 i}^{k / 3}}{T_{i}^{k}} \\
\text { s.t. } \quad T_{Y}^{2} & =c^{2} \sum_{i=1}^{10} S_{i}^{2} T_{i}^{2}
\end{aligned}
$$

with the same meaning of $f_{C}$ as in the previous example.

The mechanism can be treated as an exactly constrained linkage by locking slider $\mathrm{A}$ in the given pose. Consequently,

Table 2 Dimensions related to the output for the mechanism

\begin{tabular}{lll}
\hline Dimension & Description & $X_{0 i}$ \\
\hline$X_{1}$ & Length of BD & $r_{B}$ \\
$X_{2}$ & Length of CD & $l$ \\
$X_{3}$ & Length of OD & $h$ \\
$X_{4}$ & Length of OE & $l$ \\
$X_{5}$ & Length of CE & $h$ \\
$X_{6}$ & Length of AE & $r_{A}$ \\
$X_{7}$ & Diameter of holes & $D$ \\
$X_{8}$ & Diameter of pins & $d$ \\
$X_{9}$ & Angle CDB, radians & $\cos ^{3} \delta / r_{B} l$ \\
$X_{10}$ & Angle CEA, radians & $\cos ^{3} \delta / r_{A} h$ \\
\hline
\end{tabular}


the equivalent static model in Fig. 9a has fixed supports in $\mathrm{O}$ and $\mathrm{A}$. The external force $F$ is applied to $\mathrm{B}$ along direction OB. The free-body diagram in Fig. $9 \mathrm{~b}$ shows the forces acting on all links and pins. The equilibrium equations to each part give the following expressions for the unknown forces:

$F_{D}=F \frac{l \cos (\alpha+\delta)+r_{B} \sin \alpha}{l \cos (\alpha+\beta+\delta)}$

$F_{C H}=F_{D} \sin (\beta+\delta)-F \sin \delta$

$F_{C V}=F_{D} \cos (\beta+\delta)-F \cos \delta$

$F_{E}=\frac{F_{C H}\left[r_{A} \sin \beta+h \cos (\beta+\delta)\right]+F_{C V}\left[r_{A} \cos \beta+h \cos (\beta+\delta)\right]}{r_{A} \sin (\alpha+\beta+\delta)}$

$F_{A H}=F_{E} \cos \alpha-F_{C H}$

$F_{A V}=-F_{E} \sin \alpha+F_{C V}$

$F_{O H}=-F_{D} \sin (\beta+\delta)+F_{E} \cos \alpha$

$F_{O V}=F_{D} \cos (\beta+\delta)-F_{E} \sin \alpha$

Then, the resultants $F_{C}, F_{E}, F_{A}$, and $F_{O}$ are calculated as the vector sums of the respective components.

According to the static analogy, the sensitivities of $Y$ to the lengths are calculated from (12):

$S_{1}=\frac{F}{F} \cos \alpha=\cos \alpha$

$S_{2}=\frac{F_{C H}}{F} \cos \alpha+\frac{F_{C V}}{F} \sin \alpha$

$S_{3}=\frac{F_{D}}{F}$

$S_{4}=-\frac{F_{E}}{F}$

$S_{5}=-\frac{F_{C H}}{F} \sin (\beta+\delta)-\frac{F_{C V}}{F} \cos (\beta+\delta)$

$S_{6}=-\frac{F_{A H}}{F} \cos \beta-\frac{F_{A V}}{F} \sin \beta$

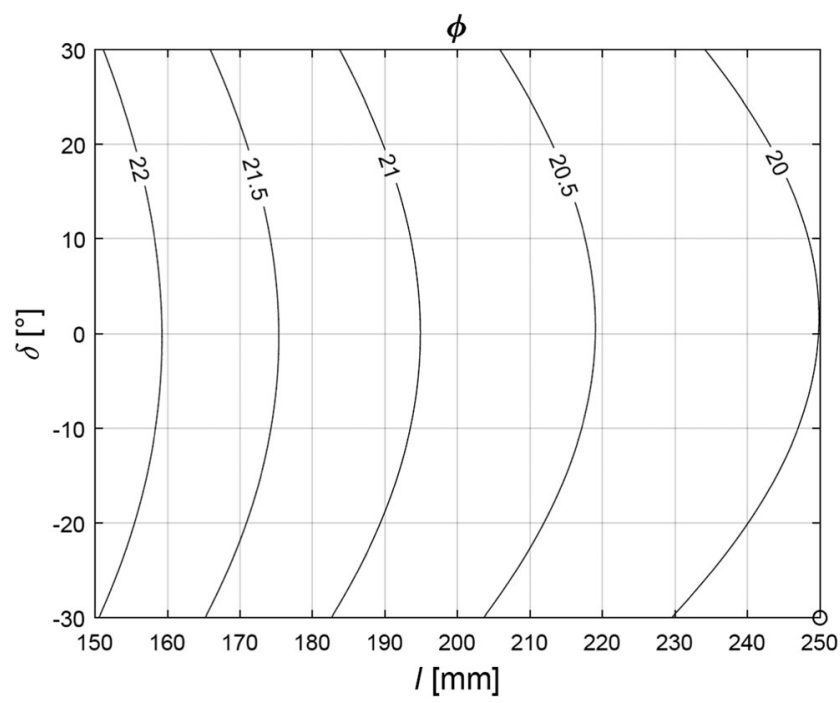

Fig. 10 Output variance for the mechanism

The sensitivities of $Y$ to the diameters are calculated by adding the contributions of the corresponding forces from (13) and (14):

$$
\begin{aligned}
& S_{7}=\frac{1}{2} \sqrt{\left(\frac{F_{A}}{F}\right)^{2}+2\left(\frac{F_{C}}{F}\right)^{2}+3\left(\frac{F_{D}}{F}\right)^{2}+3\left(\frac{F_{E}}{F}\right)^{2}} \\
& S_{8}=-\sqrt{\left(\frac{F_{A}}{F}\right)^{2}+\left(\frac{F_{C}}{F}\right)^{2}+\left(\frac{F_{D}}{F}\right)^{2}+\left(\frac{F_{E}}{F}\right)^{2}+\left(\frac{F_{O}+F_{D}+F_{E}}{F}\right)^{2}}
\end{aligned}
$$

The sensitivities of $Y$ with respect to the angles in radians are given by the bending moments at the vertices $\mathrm{D}$ and $\mathrm{E}$ of the levers, divided by the external force:

$$
\begin{aligned}
S_{9} & =-r_{B} \sin \alpha \\
S_{10} & =-r_{A}\left(\frac{F_{A H}}{F} \sin \beta-\frac{F_{A V}}{F} \cos \beta\right)
\end{aligned}
$$

Fig. 9 Equivalent static model for the mechanism: (a) constraints and external force; (b) free-body diagram

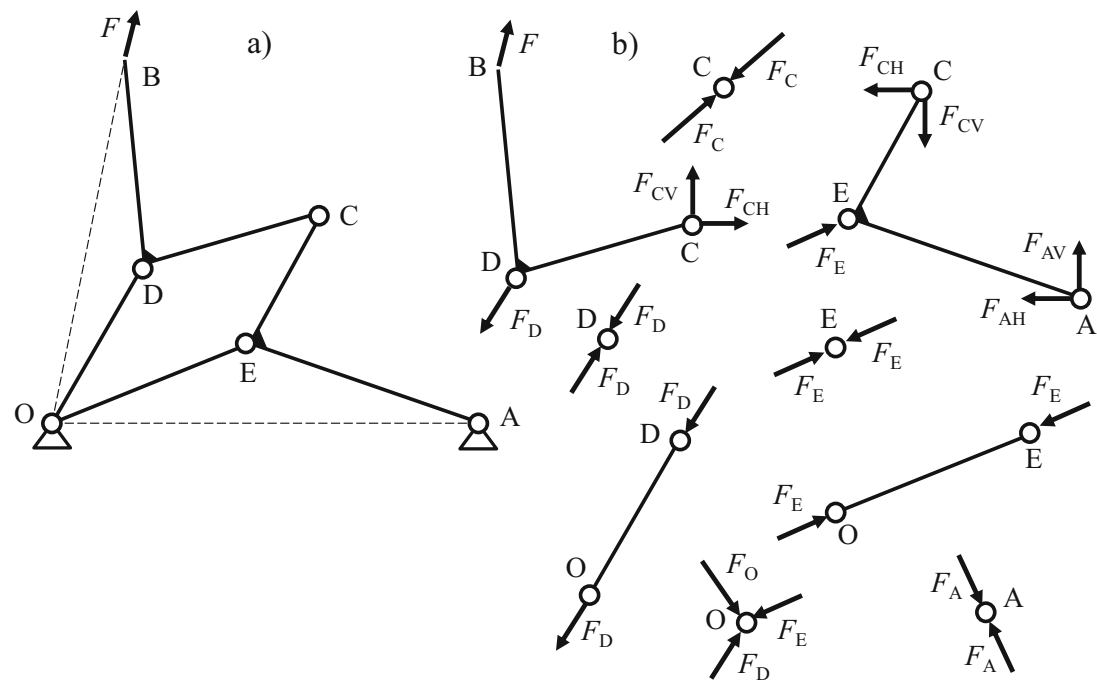


Table 3 Optimization results for the mechanism

\begin{tabular}{llll}
\hline Dimension & $X_{0 i}$ & $T_{i}$ & $\left|S_{i}\right|$ \\
\hline$X_{1}$ & $200 \mathrm{~mm}$ & $0.074 \mathrm{~mm}$ & 0.929 \\
$X_{2}$ & $250 \mathrm{~mm}$ & $0.078 \mathrm{~mm}$ & 0.886 \\
$X_{3}$ & $120 \mathrm{~mm}$ & $0.049 \mathrm{~mm}$ & 1.486 \\
$X_{4}$ & $250 \mathrm{~mm}$ & $0.048 \mathrm{~mm}$ & 1.629 \\
$X_{5}$ & $120 \mathrm{~mm}$ & $0.089 \mathrm{~mm}$ & 0.700 \\
$X_{6}$ & $150 \mathrm{~mm}$ & $0.099 \mathrm{~mm}$ & 0.629 \\
$X_{7}$ & $10 \mathrm{~mm}$ & $0.032 \mathrm{~mm}$ & 2.059 \\
$X_{8}$ & $10 \mathrm{~mm}$ & $0.021 \mathrm{~mm}$ & 3.441 \\
$X_{9}$ & $120^{\circ}$ & $0.098 \mathrm{~mm}$ & $1.296 \mathrm{~mm} /{ }^{\circ}$ \\
$X_{10}$ & $120^{\circ}$ & $0.098 \mathrm{~mm}$ & $1.296 \mathrm{~mm} /{ }^{\circ}$ \\
\hline
\end{tabular}

The output variance

$\phi(\delta, l)=\sum_{i=1}^{10} X_{0 i}^{a}\left|S_{i}\right|^{3 a}$

varies with the two independent dimensions as shown in the graph of Fig. 10. The function is decreasing with length $l$ : for any $\delta$ the optimal length is the maximum of the predefined range (from 50 to $150 \%$ of length $r_{\mathrm{B}}$ ). The function is influenced only marginally by $\delta$, with minimum values at the extremes of its range (from $30^{\circ}$ to $-30^{\circ}$ ). The point of minimum indicated in the graph corresponds to $\delta=-30^{\circ}$ and $l=$ $250 \mathrm{~mm}$, which give $h=120 \mathrm{~mm}, \alpha=21.8^{\circ}, \beta=37.8^{\circ}$, and $\gamma=60^{\circ}$ in the given pose of the mechanism.

Again, the optimal allocation of the tolerances $T_{i}(i=1, \ldots$ 10 ) is given by (11), where the nominal dimensions $X_{0 i}$ and the sensitivities $S_{i}$ have now been evaluated from the dimensions of the mechanism as listed in Table 3. The optimal tolerances are calculated assuming $T_{Y}=0.4 \mathrm{~mm}$ and $c=1.5$. Figure 11 shows a drawing of the optimized structure.

Figure 12 shows the graph of the total cost $f_{C}$ defined in (18) with the same values of $r_{A}, r_{B}, D$, and $d$. Again, the cost varies in the solution domain very similarly to the output variance. The solution found with the proposed method is

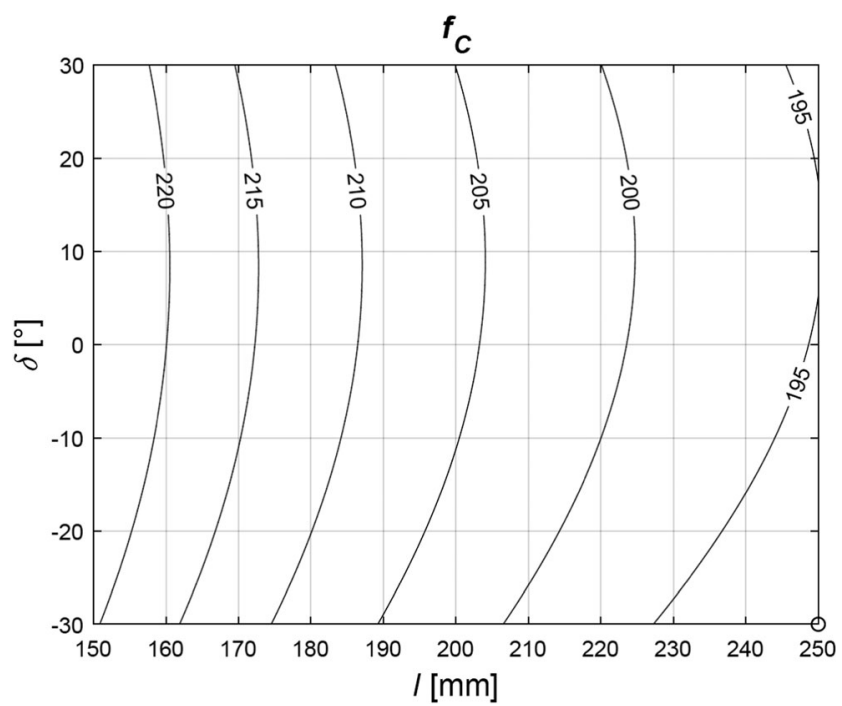

Fig. 12 Total cost for the mechanism

actually optimal with respect to cost $\left(f_{C}=190.5\right)$. Within the allowable ranges for the two independent dimensions, the cost varies in a range of about $20 \%$.

\section{Conclusions}

The paper has dealt with an extended version of tolerance allocation on planar linkages. In addition to choosing a set of minimum-cost dimensional tolerances, it seeks optimal values for the nominal dimensions in order to reduce the variation on an assembly-level dimension of interest. A linkage with optimal dimensional parameters will need wider tolerances on the parts, allowing a further cost reduction.

The concept has its roots in the robust design methodology, which aims to make the output of a system insensitive to internal or external noise factors. While most existing methods are based on experimental design, the proposed method adopts a robust optimization approach as it finds an optimal solution using mathematical models of the objective and constraints. This is feasible for mechanical assemblies (including
Fig. 11 Optimal solution for the mechanism

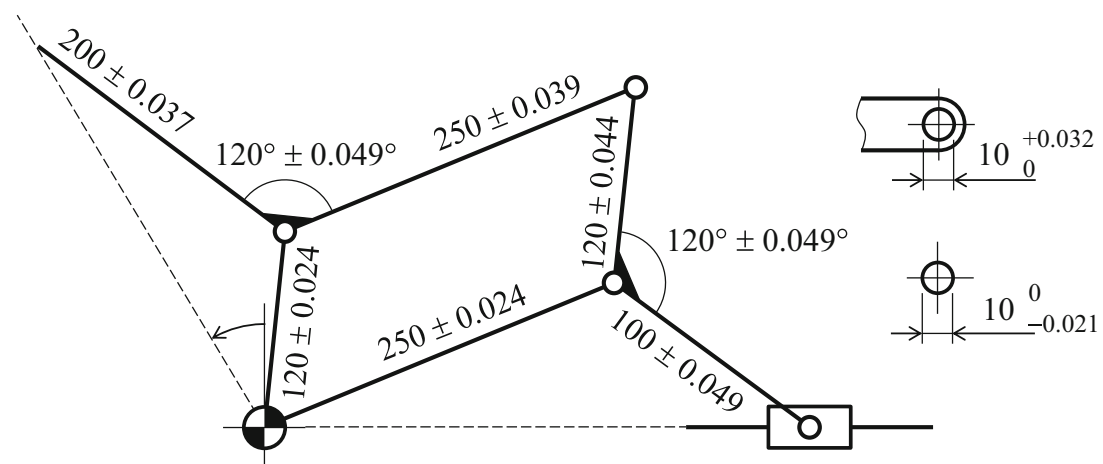


the structures and mechanisms covered in the paper) provided that the cost and the output variance have simple analytical expressions. To meet this condition, the method integrates two previous results: a static analogy for stackup calculations, and an expanded formulation of the reciprocal power costtolerance function.

The approach has potential advantages over existing methods for optimizing both dimensions and tolerances:

- Being based on optimization rather than on DOE, it is suitable to cases with many dimensions varying within wide ranges; it can easily take into account geometric closure constraints, and allows a continuous search for the optimum without the need to choose among discrete alternatives (as in Taguchi methods) or to iterate the experiment (as in RSM approaches);

- It minimizes both output variance and cost without the need to tackle the two optimization problems sequentially (with the risk of obtaining suboptimal solutions) or to balance the two objectives (with the risk of arbitrariness in the weighting coefficients).

The application of the method to two simple examples confirms that the proposed optimization strategy is able to find optimal solutions with respect to cost. It also shows that further cost reductions in the order of 10-20\% can be achieved compared to tolerance allocation with given parameters. It is expected that the simultaneous optimization of dimensions and tolerances can be even more advantageous for more complex design cases.

On the other hand, the method in its current state has some limitations, which will have to be overcome in future developments:

- The assumptions are realistic but do not cover all possible applications; further extensions may consider cases of interrelated tolerance chains, non-normal distributions of dimensions, minimization of quality loss, and geometric tolerances according to ISO and ASME standards;

- Setting up the optimization problem requires a preliminary study to reduce the solution space to a subset of independent dimensions; it remains to be seen whether general criteria can be developed to take into account geometric closure as well as additional design constraints (kinematic, structural);

- The robust optimization of a linkage is bound to a given layout of the links and joints; further cost reductions could be obtained by extending the optimization to alternative layouts, where the number and the connections of the links are also optimized along with dimensions and tolerances.

- Some calculation details are linked to the specific application context of this work (planar linkages with lower kinematic pairs); these should be generalized in order to allow the treatment of spatial linkages and generic mechanical assemblies.

Funding Open access funding provided by Politecnico di Milano within the CRUI-CARE Agreement.

Data availability The author confirms that the data supporting the findings of this study are available within the paper.

\section{Compliance with ethical standards}

Conflict of interest The author declares that he has no conflicts of interest.

Code availability Not applicable.

Open Access This article is licensed under a Creative Commons Attribution 4.0 International License, which permits use, sharing, adaptation, distribution and reproduction in any medium or format, as long as you give appropriate credit to the original author(s) and the source, provide a link to the Creative Commons licence, and indicate if changes were made. The images or other third party material in this article are included in the article's Creative Commons licence, unless indicated otherwise in a credit line to the material. If material is not included in the article's Creative Commons licence and your intended use is not permitted by statutory regulation or exceeds the permitted use, you will need to obtain permission directly from the copyright holder. To view a copy of this licence, visit http://creativecommons.org/licenses/by/4.0/.

\section{References}

1. Taguchi G, Wu Y (1979) Introduction to off-line quality control. Central Japan Quality Control Association, Nagoya

2. Taguchi G (1986) Introduction to quality engineering. UNIPUB/ Kraus International, White Plains, NY

3. Taguchi G (1987) System of experimental design: engineering methods to optimize quality and minimize cost. UNIPUB/Kraus International, White Plains, NY

4. Phadke SM (1989) Quality engineering using robust design. Prentice Hall, Englewood Cliffs, NJ

5. Acharya UH, Gijo EV, Antony J (2009) Quality engineering of a traction alternator by robust design. Proc IMechE Part B J Eng Manuf 224:297-304

6. Lin CW (2011) An application of Taguchi method on the highspeed motorized spindle system design. Proc IMechE Part C J Mech Eng Sci 225:2198-2205

7. Yip WS, To S, Wang WK (2019) Design of an optical lens for LED lighting using a hybrid principal components analysisTaguchi method. Light Res Technol 51:788-802

8. Ebro M, Howard TJ (2016) Robust design principles for reducing variation in functional performance. J Eng Des 27(1-3):75-92

9. Göhler SM, Eifler T, Howard TJ (2016) Robustness metrics: consolidating the multiple approaches to quantify robustness. ASME J Mech Des 138:111407

10. Vining GG, Myers RH (1990) Combining Taguchi and response surface philosophies: a dual response approach. J Qual Technol $22: 38-45$

11. Lucas JM (1994) How to achieve a robust process using response surface methodology. J Qual Technol 26:248-260 
12. Robinson TJ, Borror CM, Myers RH (2004) Robust parameter design: a review. Qual Reliab Eng Int 20:81-101

13. Le TH, Shin S (2018) A literature review on RSM-based robust parameter design (RPD): experimental design, estimation modeling, and optimization methods. J Korean Soc Qual Manag 46(1): 39-74

14. Wu FC, Chyu CC (2004) Optimization of robust design for multiple quality characteristics. Int J Prod Res 42(2):337-354

15. Chen W, Allen JK, Tsui KL, Mistree F (1996) A procedure for robust design: minimizing variations caused by noise factors and control factors. ASME J Mech Des 118(4):478-485

16. Kalsi M, Hacker K, Lewis K (2001) A comprehensive robust design approach for decision trade-offs in complex systems design. ASME J Mech Des 123:1-10

17. Yu JC, Ishii K (1998) Design for robustness based on manufacturing variation patterns. ASME J Mech Des 120(2):196-202

18. Allen TT, Ittiwattana W, Richardson RW, Maul GP (2001) A method for robust process design based on direct minimization of expected loss applied to arc welding. J Manuf Syst 20(5): 329-348

19. Rajagopal R, Del Castillo E (2006) A Bayesian method for robust tolerance control and parameter design. IIE Trans 38:685-697

20. Mulvey J, Vanderbei R, Zenios S (1995) Robust optimization of large-scale systems. Oper Res 43(2):264-281

21. Otto KN, Antonsson EK (1993) Extensions to the Taguchi method of product design. ASME J Mech Des 115(1):5-13

22. Park GJ, Lee TH, Lee KH, Hwang KH (2006) Robust design: an overview. AIAA J 44(1):181-191

23. Beyer HG, Sendhoff B (2007) Robust optimization: a comprehensive review. Comput Methods Appl Mech Eng 196:3190-3218

24. Parkinson DB (1997) Robust design by variability optimization. Qual Reliab Eng Int 13:97-102

25. Parkinson DB (2002) An investigation of noise factor effects in parameter design. Qual Reliab Eng Int 18:37-43

26. Parkinson DB (2008) Function variability optimization in parameter design. Qual Technol Quant Manag 5(3):263-270

27. Lu XJ, Li HX (2009) Perturbation theory based robust design under model uncertainty. ASME J Mech Des 131:111006

28. Cao Y, Liu T, Yang J (2018) A comprehensive review of tolerance analysis models. Int J Adv Manuf Technol 97:3055-3085

29. Chase KW, Greenwood WH (1988) Design issues in mechanical tolerance analysis. ASME Manuf Rev 1(1):50-59

30. Chase KW (1999) Minimum-cost tolerance allocation. In: Drake PJ (ed) Dimensioning and tolerancing handbook. Mc-Graw-Hill, New York

31. Hallmann M, Schleich B, Wartzack S (2020) From tolerance allocation to tolerance-cost optimization: a comprehensive literature review. Int J Adv Manuf Technol 107(11-12):4859-4912

32. Singh PK, Jain PK, Jain SC (2009) Important issues in tolerance design of mechanical assemblies. Part 2: tolerance synthesis. Proc IMechE Part B J Eng Manuf 223:1249-1287

33. Karmakar S, Maiti J (2012) A review on dimensional tolerance synthesis: paradigm shift from product to process. Assem Autom 32(4):373-388

34. Spotts MF (1973) Allocation of tolerances to minimize cost of assembly. ASME J Eng Ind 95:762-764

35. Creveling CM (1997) Tolerance design: a handbook for developing optimal specifications. Addison-Wesley, Reading

36. D'Errico JR, Zaino NA (1988) Statistical tolerancing using a modification of Taguchi's method. Technometrics 30(4):397-405

37. Gerth RJ, Islam Z (1998) Towards a designed experiments approach to tolerance design. In: Geometric design tolerancing: theories, standards and applications. Springer, Boston, pp 337-345

38. Bisgaard S (1997) Designing experiments for tolerancing assembled products. Technometrics 39(2):142-152
39. Feng CX, Kusiak A (2000) Robust tolerance synthesis with the design of experiments approach. ASME J Manuf Sci Eng 122: 520-528

40. Gerth RJ, Klonaris P, Pfeiffer T (1999) Cost tolerance sensitivity analysis for concurrent engineering design support. In: Global consistency of tolerances. Springer, Dordrecht, pp 313-324

41. Gerth RJ, Pfeiffer T (2000) Minimum cost tolerancing under uncertain cost estimates. IIE Trans 32:493-503

42. Gerth RJ, Pfeiffer T, Oehme O (2000) Early cost tolerance sensitivity analysis for inspection planning. Hum Factors Ergon Manuf 10(3):309-329

43. Schmitt R, Behrens C (2007) A statistical method for analyses of cost- and risk-optimal tolerance allocations based on assured input data. Proc CIRP Conf Computer-Aided Tolerancing, Erlangen, Germany

44. Jeang A (1997) An approach of tolerance design for quality improvement and cost reduction. Int J Prod Res 35(5):1193-1211

45. Naidu NVR (2008) Mathematical model for quality cost optimization. Robot Comput Integr Manuf 24:811-815

46. Moskowitz H, Plante R, Duffy J (2001) Multivariate tolerance design using quality loss. IIE Trans 33:437-448

47. Plante R (2002) Multivariate tolerance design for a quadratic design parameter model. IIE Trans 34:565-571

48. Jeang A, Leu E (1999) Robust tolerance design by computer experiment. Int J Prod Res 37(9):1949-1961

49. Lööf J, Hermansson T, Söderberg R (2007) An efficient solution to the discrete least-cost tolerance allocation problem with general loss functions. In: Davidson JK (ed) Models for computer-aided tolerancing in design and manufacturing. Springer, Dordrecht, pp $115-124$

50. Jeang A (1999) Optimal tolerance design by response surface methodology. Int J Prod Res 37(14):3275-3288

51. Jeang A (2001) Computer-aided tolerance synthesis with statistical method and optimization techniques. Qual Reliab Eng Int 17: 131-139

52. Jeang A, Tsai SW, Li HC, Hsieh CK (2002) A computer model for time-based tolerance design with response surface methodology. Int J Comput Integr Manuf 15(2):97-108

53. Li CC, Kao C, Chen SP (1998) Robust tolerance allocation using stochastic programming. Eng Optim 30:333-350

54. Kao C, Li CC, Chen SP (2000) Tolerance allocation via simulation embedded sequential quadratic programming. Int J Prod Res 38(17):4345-4355

55. Savage GJ, Tong D, Carr SM (2006) Optimal mean and tolerance allocation using conformance-based design. Qual Reliab Eng Int 22:445-472

56. Forouraghi B (2002) Worst-case tolerance design and quality assurance via genetic algorithms. J Optim Theory Appl 113(2):251268

57. Pandya G, Lehtihet EA, Cavalier TM (2002) Tolerance design of datum systems. Int J Prod Res 40(4):783-807

58. Zhang C, Wang HP (1998) Robust design of assembly and machining tolerance allocations. IIE Trans 30(1):17-29

59. Ji S, Li X, Du X (2000) Tolerance synthesis using second-order fuzzy comprehensive evaluation and genetic algorithm. Int J Prod Res 38(15):3471-3483

60. Ji S, Li X, Ma Y, Cai H (2000) Optimal tolerance allocation based on fuzzy comprehensive evaluation and genetic algorithm. Int $\mathbf{J}$ Adv Manuf Technol 16:461-468

61. Huang YM, Shiau CS (2006) Optimal tolerance allocation for a sliding vane compressor. ASME J Mech Des 128:98-107

62. Lyu N, Shimura A, Saitou K (2006) Optimal tolerance allocation of automotive pneumatic control valves based on product and process simulations. Proc ASME Int Design Engineering Technical Conf., Philadelphia, PA 
63. Geetha K, Ravindran D, Siva Kumar M, Islam MN (2013) Multiobjective optimization for optimum tolerance synthesis with process and machine selection using a genetic algorithm. Int J Adv Manuf Technol 67:2439-2457

64. Xing Y, Wang Y (2013) Design and optimisation of assembly technique for auto-body components. Int J Prod Res 51(22): 6515-6533

65. Huang B, Du X (2007) Analytical robustness assessment for robust design. Struct Multidiscip Optim 34:123-137

66. Zhang Y, Li M (2018) Robust tolerance optimization for internal combustion engines under parameter and model uncertainties considering metamodeling uncertainty from Gaussian processes. ASME J Comput Inf Sci Eng 18:041011

67. Jeang A (2001) Combined parameter and tolerance design optimization with quality and cost. Int J Prod Res 39(5):923-952

68. Jeang A, Chung CP, Hsieh CK (2007) Simultaneous process mean and process tolerance determination with asymmetrical loss function. Int J Adv Manuf Technol 31:694-704

69. Jeang A, Chung CP (2008) Process mean, process tolerance, and use time determination for product life application under deteriorating process. Int J Adv Manuf Technol 36:97-113

70. Jeang A, Chang CL (2002) Concurrent optimisation of parameter and tolerance design via computer simulation and statistical method. Int J Adv Manuf Technol 19:432-441

71. Jeang A, Chen TK, Hwan CL (2002) A statistical dimension and tolerance design for mechanical assembly under thermal impact. Int J Adv Manuf Technol 20:907-915

72. Jeang A (2003) Robust computer-aided parameter and tolerance determination for an electronic circuit design. Int J Prod Res 41(5): 883-895

73. Jeang A (2008) Combined parameter and tolerance design for quality via computer experiment: a design for thermoelectric microactuator. IEEE Trans Electron Packag Manuf 31(3):192201

74. Jeang A, Liang F, Chung CP (2007) Robust product development for multiple quality characteristics using computer experiments and an optimization technique. Int J Prod Res 46(12):3415-3439

75. Takai S, Jikar VK, Ragsdell KM (2011) An approach toward integrating top-down and bottom-up product concept and design selection. ASME J Mech Des 133:071007

76. Hu J, Xiong G (2005) Concurrent design of a geometric parameter and tolerance for assembly and cost. Int J Prod Res 43(2):267-293

77. Hu J, Peng Y (2007) Tolerance modelling and robust design for concurrent engineering. Proc IMechE Part C J Mech Eng Sci 221: $455-465$

78. Hung TC, Chan KY (2013) Multi-objective design and tolerance allocation for single and multi-level systems. J Intell Manuf 24: 559-573

79. Gu XG, Tong WT, Han MM, Wang Y, Wang ZY (2019) Parameter and tolerance economic design for multivariate quality characteristics based on the modified process capability index with individual observations. IEEE Access 7:59249-59262

80. Prabhaharan G, Asokan P, Rajendran S (2005) Sensitivity-based conceptual design and tolerance allocation using the continuous ants colony algorithm (CACO). Int J Adv Manuf Technol 25:516526

81. Cho BR, Kim YJ, Kimbler DL, Phillips MD (2000) An integrated joint optimization procedure for robust and tolerance design. Int J Prod Res 38(10):2309-2325

82. Shin S, Kongsuwon P, Cho BR (2010) Development of the parametric tolerance modeling and optimization schemes and costeffective solutions. Eur J Oper Res 207:1728-1741
83. Vignesh Kumar D, Ravindran D, Lenin N, Siva Kumar M (2019) Tolerance allocation of complex assembly with nominal dimension selection using Artificial Bee Colony algorithm. Proc IMechE Part C J Mech Eng Sci 233(1):18-38

84. Zhang J, Li SP, Bao NS, Zhang GJ, Xue DY, Gu PH (2010) A robust design approach to determination of tolerances of mechanical products. CIRP Ann Manuf Technol 59:195-198

85. Jeang A, Chen T, Li HC, Liang F (2007) Simultaneous process mean and process tolerance determination with adjustment and compensation for precision manufacturing process. Int J Adv Manuf Technol 33:1159-1172

86. Schueller GI, Jensen HA (2008) Computational methods in optimization considering uncertainties: an overview. Comput Methods Appl Mech Eng 198:2-13

87. Lee KH, Park GJ (2001) Robust optimization considering tolerances of design variables. Comput Struct 79:77-86

88. Doltsinis I, Kang Z (2004) Robust design of structures using optimization methods. Comput Methods Appl Mech Eng 193:22212237

89. Kang Z, Bai S (2013) On robust design optimization of truss structures with bounded uncertainties. Struct Multidiscip Optim 47:699-714

90. Kota S, Chiou SJ (1993) Use of orthogonal arrays in mechanism synthesis. Mech Mach Theory 28(6):777-794

91. Da Lio M (1997) Robust design of linkages: synthesis by solving non-linear optimization problems. Mech Mach Theory 32(8):921932

92. Du X, Venigella PK, Liu D (2009) Robust mechanism synthesis with random and interval variables. Mech Mach Theory 44:13211337

93. Zhang J, Wang J, Du X (2011) Time-dependent probabilistic synthesis for function generator mechanisms. Mech Mach Theory 46: $1236-1250$

94. Luo K, Wang J, Du X (2012) Robust mechanism synthesis with truncated dimension variables and interval clearance variables. Mech Mach Theory 57:71-83

95. Shi Z, Yang X, Yang W, Cheng Q (2005) Robust synthesis of path generating linkages. Mech Mach Theory 40:45-54

96. Arenbeck H, Missoum S, Basudhar A, Nikravesh P (2010) Reliability-based optimal design and tolerancing for multibody systems using explicit design space decomposition. ASME J Mech Des 132:021010

97. Sutherland GH, Roth B (1975) Mechanism design: accounting for manufacturing tolerances and costs in function generating problems. ASME J Eng Ind 97(1):283-286

98. Choi JH, Lee SJ, Choi DH (1998) Tolerance optimization for mechanisms with lubricated joints. Multibody Sys Dyn 2:145168

99. Rao SS, Wu W (2005) Optimum tolerance allocation in mechanical assemblies using an interval method. Eng Optim 37(3):237257

100. Wu W, Rao SS (2007) Uncertainty analysis and allocation of joint tolerances in robot manipulators based on interval analysis. Reliab Eng Syst Saf 92:54-64

101. Rout BK, Mittal RK (2007) Tolerance design of manipulator parameters using design of experiment approach. Struct Multidiscip Optim 34:445-462

102. Rout BK, Mittal RK (2008) Parametric design optimization of 2DOF R-R planar manipulator: a design of experiment approach. Robot Comput Integr Manuf 24:239-248

103. Huang X, Zhang Y (2010) Robust tolerance design for function generation mechanisms with joint clearances. Mech Mach Theory 45:1286-1297 
104. Chen FC, Tzeng YF, Chen WR, Hsu MH (2009) The use of the Taguchi method and principal component analysis for the sensitivity analysis of a dual-purpose six-bar mechanism. Proc IMechE Part C J Mech Eng Sci 223:733-741

105. Li JG, Ding J, Yao YX, Fang HG (2015) A new accuracy design for a 6-dof docking mechanism. Proc IMechE Part C J Mech Eng Sci 229(18):3473-3483

106. Huang T, Bai P, Mei J, Chetwynd DG (2016) Tolerance design and kinematic calibration of a 4-DOF pick-and-place parallel robot. J Mech Robot 8(6):061018

107. Niu P, Cheng Q, Chang W, Song X, Li Y (2020) Sensitivity analysis of machining accuracy reliability considering partial correlation of geometric errors for horizontal machining center. Proc IMechE Part B J Eng Manuf DOI: https://doi.org/10.1177/ 0954405420958843

108. Zhu J, Ting KL (2001) Performance distribution analysis and robust design. ASME J Mech Des 123:11-17
109. Caro S, Bennis F, Wenger P (2005) Tolerance synthesis of mechanisms: a robust design approach. ASME J Mech Des 127:86-94

110. Armillotta A (2020) Selection of parameters in cost-tolerance functions: review and approach. Int J Adv Manuf Technol 108: 167-182

111. Evans DH (1975) Statistical tolerancing: the state of the art. Part III: shifts and drifts. J Qual Technol 7(2):72-76

112. Armillotta A (2014) A static analogy for 2D tolerance analysis. Assem Autom 34(2):182-191

113. Armillotta A (2015) Force analysis as a support to computer-aided tolerancing of planar linkages. Mech Mach Theory 93:11-25

114. Thang ND (2017) 2700 animated mechanical mechanisms. https:// youtu.be/yPJMSe7dXLM

Publisher's note Springer Nature remains neutral with regard to jurisdictional claims in published maps and institutional affiliations. 